\title{
One-Lung Ventilation with High Tidal Volumes and Zero Positive End-Expiratory Pressure Is Injurious in the Isolated Rabbit Lung Model
}

\author{
Marcelo Gama de Abreu, PhD, MD, MSc, Manuel Heintz, MD, Axel Heller, PhD, MD, \\ Roswitha Széchényi, Detlev Michael Albrecht, MD, PhD, and Thea Koch, MD, PhD \\ Clinic of Anesthesiology and Intensive Care Medicine, University Clinic Carl Gustav Carus, Technical University \\ Dresden, Dresden, Germany
}

\begin{abstract}
We tested the hypothesis that one-lung ventilation (OLV) with high tidal volumes (VT) and zero positive endexpiratory pressure (PEEP) may lead to ventilatorinduced lung injury. In an isolated, perfused rabbit lung model, VT and PEEP were set to avoid lung collapse and overdistension in both lungs, resulting in a straight pressure-time (P-vs-t) curve during constant flow. Animals were randomized to (a) nonprotective OLV (left lung) $(n=6)$, with VT values as high as before randomization and zero PEEP; (b) protective OLV (left lung) $(n=6)$, with $50 \%$ reduction of $\mathrm{VT}$ and maintenance of PEEP as before randomization; and (c) control group $(n=6)$, with ventilation of two lungs as before randomization. The nonprotective OLV was associated with significantly
\end{abstract}

smaller degrees of collapse and overdistension in the ventilated lung $(P<0.001)$. Peak inspiratory pressure values were higher in the nonprotective OLV group $(P<0.001)$ and increased progressively throughout the observation period $(P<0.01)$. The mean pulmonary artery pressure and lung weight gain values, as well as the concentration of thromboxane $\mathrm{B}_{2}$, were comparatively higher in the nonprotective OLV group $(P<0.05)$. A ventilatory strategy with VT values as high as those used in the clinical setting and zero PEEP leads to ventilator-induced lung injury in this model of OLV, but this can be minimized with VT and PEEP values set to avoid lung overdistension and collapse.
0 ne-lung ventilation (OLV) may be required to achieve an optimal surgical field or to protect one lung from major bleeding or infection in the opposite side. Unfortunately, this procedure may be associated with an important impairment in gas exchange, particularly in patients with previous lung disease (1). Therefore, maintenance of adequate arterial oxygenation and $\mathrm{CO}_{2}$ elimination represents the major challenge of the ventilatory strategy during OLV. The traditional approach to achieve these goals includes the use of tidal volumes (VT) almost as high as during ventilation of 2 lungs (i.e., 10 to $12 \mathrm{~mL} / \mathrm{kg}$ ) and zero positive end-expiratory pressure (PEEP), with the inspiratory oxygen fraction being titrated as necessary (2). The rationale for this approach is that

\section{Supported by departmental funds.}

Accepted for publication September 18, 2002.

Address correspondence and reprint requests to Marcelo Gama de Abreu, MD, MSc, Clinic of Anesthesiology and Intensive Care Medicine, University Clinic Carl Gustav Carus, Technical University Dresden, Fetscherstr. 74, 01307 Dresden, Germany. Address e-mail to mgabreu@aol.com. the use of relatively high $\mathrm{VT}_{\mathrm{T}}$ values is useful to increase the alveolar ventilation, thereby improving both oxygenation and $\mathrm{CO}_{2}$ elimination (2). Low PEEP values should avoid the pulmonary blood shifting from the ventilated to the nonventilated lung, a phenomenon that can counteract the protective effect of hypoxic vasoconstriction $(3,4)$. Because the priority of this approach requires the adequacy of gas exchange and because OLV is usually limited in time, less attention is paid to the potential harmful effects of the ventilatory strategy to the lung parenchyma.

Different studies have shown that mechanical ventilation may initiate or worsen lung injury $(5,6)$. Also, it has been demonstrated that cyclic collapse and reopening of alveoli at lower PEEP levels may lead to the development of shear-stress forces and lung injury (7). Those studies, however, were performed with ventilation of both lungs. The OLV situation may differ from the previous settings in two respects: first, the ventilated lung is usually not previously injured. Second, extreme overdistension, as used to achieve lung injury in other studies (5), is usually not present, but the distribution of ventilation could theoretically lead to similar degrees of mechanical stress in some lung 
zones. However, no study has addressed the development of ventilator-induced lung injury (VILI) during OLV and the use of protective respiratory strategies under such conditions.

In this work, we investigated whether a nonprotective mechanical ventilatory strategy for OLV with high Vт values and zero PEEP may cause VILI in the isolated rabbit lung model. Also, we tested the hypothesis that the titration of VT and PEEP to avoid lung overdistension and collapse would have a protective effect, minimizing VILI during OLV.

\section{Methods}

The protocol of this study was approved by the Ethics Committee for Experimental Research of the Carl Gustav Carus University Hospital, Dresden, Germany, and is in accordance with the National Institutes of Health guidelines for animal use. The preparation of the isolated, perfused rabbit lung model has been described in detail by our group elsewhere (8). Briefly, female rabbits (Orticolagus caniculus) weighing 1.6 to $2.6 \mathrm{~kg}$ were anesthetized with ketamine $50 \mathrm{mg} / \mathrm{kg}$ (CuraMED, Karlsruhe, Germany) and xylazine hydrochloride $4 \mathrm{mg} / \mathrm{kg}$ (Bayer, Leverkusen, Germany) after cannulation of the auricular vein. Anticoagulation was achieved by means of IV administration of heparin $1000 \mathrm{U} / \mathrm{kg}$ (Liquemin; Hoffman-La Roche, Grenzach-Wyhlen, Germany). After pretracheal infiltration with $8 \mathrm{~mL}$ of lidocaine hydrochloride $1 \%$ (Jenapharm, Jena, Germany), a tracheotomy was performed, and the trachea was cannulated by using a $10-\mathrm{cm}$-long catheter with a diameter of $0.4 \mathrm{~cm}$ (endotracheal tube) (B. Braun, Melsungen, Germany). Animals had the lungs ventilated with room air by use of the Small Animal Ventilator KTR-4 (Hugo Sachs Elektronik $\mathrm{GmbH}$, March, Germany). This mechanical ventilator was chosen because it delivers practically constant inspiratory flow rates in the range of 100 to $1500 \mathrm{~mL} / \mathrm{min}( \pm 2 \%$ variation of flow rate set on the device). The initial ventilator settings were $\mathrm{VT}_{\mathrm{T}}=$ $8 \mathrm{~mL} / \mathrm{kg}$, respiratory frequency $=30$ breaths $/ \mathrm{min}$, PEEP $=1 \mathrm{~cm} \mathrm{H}_{2} \mathrm{O}$, and inspiratory-expiratory ratio = 1:1. PEEP was set by using an external water column connected to the expiratory port of the ventilator. Auto-PEEP was measured at the end of a 3-s endexpiratory occlusion, and total PEEP was calculated as auto-PEEP plus external PEEP.

After a median sternotomy, the pulmonary artery was cannulated, and the heart was opened to permit the exsanguination of the lungs with Krebs-Henseleit hydroxyethyl starch buffer solution (perfusate), which was pumped with a roller pump at $50 \mathrm{~mL} / \mathrm{min}$ (Masterflex L/S; Cole-Parmer, Mfg. Barnant, Barrington, IL). The lungs and trachea were carefully dissected, removed en bloc, and suspended from a weight transducer (Hottinger Baldwin Meßtechnik, Darmstadt,
Germany) in a temperature-controlled $\left(37^{\circ} \mathrm{C}\right)$ doublewalled chamber. The lung perfusate dropping from the lungs into the chamber was collected into a reservoir and redirected to the roller pump as a closed recirculation system. The total volume of perfusate in the system was $200 \mathrm{~mL}$. The temperature of the perfusate was maintained at $37^{\circ} \mathrm{C}$ with a water bath, and the $\mathrm{pH}$ was maintained between 7.35 and 7.45 by means of the inflow of $\mathrm{CO}_{2}$ into the inspiratory air, achieving a concentration of approximately $4 \% \mathrm{CO}_{2}$. The perfusate flow rate was increased to $100 \mathrm{~mL} / \mathrm{min}$, and the whole volume was exchanged two times before measurements began.

Mean pulmonary artery pressure (MPAP) was monitored continuously by means of a pressure difference transducer with the CMS Monitor (Agilent Technologies, Böblingen, Germany). The MPAP was zeroreferenced at the hilus height. Because the perfusion was held constant, alterations of the perfusion pressure were directly related to the total pulmonary vascular resistance. The weight transducer was also connected to the CMS Monitor, and both weight and MPAP were recorded each minute by means of a microcomputer. Weight gain (WG) was defined as the weight of the trachea-lung bloc measured in a given moment minus the weight measured at the beginning of the experiment.

Airway opening pressure was monitored at the connection between the intratracheal catheter and the Y-piece of the mechanical ventilator by using a differential pressure transducer referenced to the atmospheric pressure (PasCal; Hoffrichter $\mathrm{GmbH}$, Schwerin, Germany). Because the chamber containing the trachea-lung bloc was open to the atmosphere, airway opening pressure was identical to the transpulmonary pressure $\left(\mathrm{P}_{\mathrm{L}}\right)$. The $\mathrm{P}_{\mathrm{L}}$ signal was digitized at $200 \mathrm{~Hz}$ by an analog-digital board (DAQ-Pad 1200; National Instruments, Austin, TX) with 10 bits and acquired by a laptop by using a special routine developed for LabView (National Instruments).

Dynamic pressure-time (P-vs-t) curves were recorded during constant flow inflation over three consecutive respiratory cycles. The relationship between pressure and time can be described according to a power equation (9):

$$
P_{\mathrm{L}}=a \times t^{b}+c
$$

where coefficient $a$ is a constant that represents the slope of the P-vs-t relation at $t=1$, and coefficient $c$ is the pressure at $t=0$. The coefficient $b$ is a dimensionless constant that describes the concavity of the P-vs-t curve. For $b$ values $>1$, the dynamic P-vs-t curve has an upward concavity, indicating that the compliance decreases with time, as, for instance, when the lungs are being overdistended. For $b$ values $<1$, the dynamic P-vs-t curve has a downward concavity, indicating 
that the compliance increases with time, as, for instance, when the lungs are being inflated from collapse. For $b=1$, the dynamic P-vs-t curve is a straight line, indicating a constant lung compliance without collapse or overdistension. Unfortunately, Equation 1 cannot be used to describe a P-vs-t curve with concavities in both the lower and upper ranges (sigmoidal shape) appropriately (10). Therefore, in this work, we divided the P-vs-t curve into two portions. The first lower third and the upper two thirds of the dynamic P-vs-t curve were described according to Equations 2 and 3, respectively. These ranges were arbitrarily defined after careful analysis of the P-vs-t curve.

$$
\begin{gathered}
P_{\text {L.lower }}=a_{\text {lower }} \times t^{b}{ }_{\text {lower }}+c_{\text {lower }} \\
P_{\text {L,upper }}=a_{\text {upper }} \times t^{b}{ }_{\text {upper }}+c_{\text {upper }}
\end{gathered}
$$

where the coefficients $b_{\text {lower }}$ and $b_{\text {upper }}$ describe the concavity of the lower and upper portions of the P-vs- $t$ curve, respectively, in the same way as described for Equation 1.

The P-vs-t curves were processed off-line to identify the beginning and end of inspiration. Because the $\mathrm{P}_{\mathrm{L}}$ increase has a characteristic spike when the inspiratory flow starts, flow curves were not necessary to identify the beginning of inspiration. To ensure that on- and off-flow transients generated by the mechanical ventilator did not skew the results, the first and last $50 \mathrm{~ms}$ of the inspiratory cycle were excluded from the analysis. These values have been derived from preliminary experiments and are virtually the same as those used by Ranieri et al. (10) in a similar procedure. The curve-fitting procedures for the lower and upper portions of the P-vs-t curve were performed with the remaining data, and $b_{\text {lower }}$ and $b_{\text {upper }}$ were calculated according to the experimental protocol.

Perfusate samples were taken with 2-mL syringes containing $13.6 \mu \mathrm{g}$ of diclofenac (Rewodina; ASTA Medica AWD, Frankfurt, Germany) and immediately centrifuged at $14,000 \mathrm{U} / \mathrm{min}$ for $10 \mathrm{~min}$. By using a calibrated pipette, $1000 \mu \mathrm{L}$ of the samples was drawn and frozen at $-20^{\circ} \mathrm{C}$. Afterward, samples were allowed to thaw, and thromboxane $\mathrm{B}_{2}\left(\mathrm{TXB}_{2}\right)$ concentrations were measured in a blinded fashion with enzyme-linked immunosorbent assay (Institute of Biochemistry, University Clinic Carl Gustav Carus, Dresden, Germany).

Initially, PEEP was increased until a straight line was achieved in the lower part of the dynamic P-vs- $t$ curve $\left(b_{\text {lower }} \approx 1\right)$. After that, a sustained inflation with $30 \mathrm{~cm} \mathrm{H}_{2} \mathrm{O}$ was performed for $30 \mathrm{~s}$ (recruitment maneuver), and Vт was set at 6 to $8 \mathrm{~mL} / \mathrm{kg}$ to obtain a straight line also in the upper portion of the dynamic P-vs-t curve $\left(b_{\text {upper }} \approx 1\right)$. All other ventilator settings were kept constant, and the lungs were randomized to one of three groups:
1. Nonprotective OLV $(n=6)$. In this group, the endotracheal tube was carefully advanced into the left main bronchus until the left lung could be isolated. Major attention was paid to obtain ventilation of both the left upper and lower lobes without ventilating any part of the right lung. The external PEEP was set at zero, and VT was kept at the same level preceding OLV. Recruitment maneuvers were performed every $30 \mathrm{~min}$ only for the purpose of checking for respiratory system leaks.

2. Protective OLV $(n=6)$. Isolation of the left lung was performed in the same way as described above. PEEP was kept at the same level preceding OLV, and Vт was reduced by $50 \%$. In addition, recruitment maneuvers of the left lung were performed every $10 \mathrm{~min}$.

3. Control group $(n=6)$. In this group, both lungs were protectively ventilated, and the mechanical ventilator settings were kept constant. Recruitment maneuvers were performed every $10 \mathrm{~min}$.

The entire procedure to achieve adequate ventilation of one isolated lung lasted approximately $5 \mathrm{~min}$. Animals were excluded from the study if 1 ) an air leak was identified during recruitment procedures, 2) ventilation of the right lung was observed at any time after the beginning of OLV, or 3) ventilation of the upper left lobe was absent.

Measurements of respiratory mechanics were performed before randomization (baseline) and at 0, 60, and $90 \mathrm{~min}$ after OLV ventilation was established or under two-lung ventilation (control group). To compensate for the delay needed to achieve OLV in the other groups, the first measurement after randomization in the control group (time 0 ) was also delayed by $5 \mathrm{~min}$. Perfusate samples were drawn at baseline and at $0,30,60$, and $90 \mathrm{~min}$ in all groups.

All results are expressed as mean \pm SEM. Changes in ventilatory settings were assessed with paired Student's $t$-tests. Comparison among groups was performed with two-way analysis of variance with twoway entry (group and time; general linear model). Multiple comparisons were performed with the Student-Newman-Keuls correction. One-way analysis of variance was used where appropriate. A $P$ value $<0.05$ was considered to be statistically significant in all tests performed.

\section{Results}

Six experiments were excluded because of the development of air leaks after randomization (three in the nonprotective OLV group, two in the protective OLV group, and one in the control group), three because of ventilation of the contralateral lung (two in the nonprotective OLV group and one in the protective OLV 
Table 1. Ventilator Settings of the Experimental Groups

\begin{tabular}{llccccc}
\hline $\begin{array}{c}\text { Experimental } \\
\text { group }\end{array}$ & Phase & $\mathrm{V}_{\mathrm{T}}(\mathrm{mL})$ & $\begin{array}{c}\mathrm{RR} \\
\text { (breaths/min) }\end{array}$ & $\mathrm{I} / \mathrm{E}$ & $\begin{array}{c}\text { PIP } \\
\left(\mathrm{cm} \mathrm{H} \mathrm{H}_{2} \mathrm{O}\right)\end{array}$ & $\begin{array}{c}\text { PEEP } \\
\left(\mathrm{cm} \mathrm{H} \mathrm{H}_{2} \mathrm{O}\right)\end{array}$ \\
\hline Control & Pre rand & $7.7 \pm 0.9$ & 30 & $1: 1$ & $5.1 \pm 0.1$ & $2.1 \pm 0.2$ \\
& Post rand & $7.9 \pm 0.7$ & 30 & $1: 1$ & $5.1 \pm 0.1$ & $2.1 \pm 0.2$ \\
OLV prot & Pre rand & $8.2 \pm 0.4$ & 30 & $1: 1$ & $4.9 \pm 0.2$ & $2.2 \pm 0.2$ \\
& Post rand & $4.1 \pm 0.3^{*}$ & 30 & $1: 1$ & $4.8 \pm 0.2$ & $2.1 \pm 0.2$ \\
OLV nonprot & Pre rand & $8.2 \pm 0.4$ & 30 & $1: 1$ & $5.1 \pm 0.2$ & $2.1 \pm 0.2$ \\
& Post rand & $8.3 \pm 0.4$ & 30 & $1: 1$ & $13.5 \pm 0.3^{*}$ & $0.4 \pm 0.2^{*}$ \\
\hline
\end{tabular}

Values are mean \pm sEM.

OLV prot = protective one-lung ventilation; OLV nonprot = nonprotective one-lung ventilation; pre rand = prerandomization; post rand = postrandomization; $\mathrm{V}_{T}$ = tidal volume; $\mathrm{RR}=$ respiratory rate; $\mathrm{I} / \mathrm{E}=$ inspiratory-expiratory ratio; $\mathrm{PIP}=$ peak inspiratory pressure; $\mathrm{PEEP}=$ total positive end-expiratory pressure (auto PEEP + external PEEP).

${ }^{*} P<0.001$ versus prerandomization. There were no significant differences in $\mathrm{V}_{\mathrm{T}}, \mathrm{PEEP}$, or PIP among groups in the prerandomization phase.

group), and two because of absence of ventilation of the upper left lobe (protective OLV group). Animals that replaced the excluded experiments were also randomized. No differences among groups with respect to animal weights were observed $(2.0 \pm 0.2 \mathrm{~kg})$. Table 1 summarizes the ventilatory data for the three groups in the study immediately before randomization and after adjustments of the ventilatory settings (pre- and postrandomization phases, respectively).

Figure 1 shows typical P-vs-t curves, obtained from each of the three groups in the study immediately after randomization. In the nonprotective OLV group (Fig. 1, top), the P-vs-t curve shows a lower and an upper concavity, which are associated with a progressive increase of compliance at the beginning of inspiration and a progressive decrease of compliance at the end of inspiration. In the protective OLV and control groups (Fig. 1, middle and bottom, respectively), the P-vs-t curves are straight lines, indicating an approximately constant compliance over the entire inspiration.

Values of the coefficients $b_{\text {lower }}$ and $b_{\text {upper }}$ for the three groups of experiments are presented in Figure 2. For all groups, the P-vs-t relation at the lower part of the curve was linear at baseline, indicating that the compliance was constant in the lower VT range used before randomization. In the nonprotective OLV group, the lower part of the P-vs-t curve showed an downward concavity, indicating a progressive increase in compliance at the lower Vт range; values of $b_{\text {lower }}$ were situated between 0.34 and 0.86 throughout the experiments. In the protective OLV group, the lower part of the P-vs-t curve was linear, indicating a constant compliance at the beginning of inspiration; values of $b_{\text {lower }}$ were situated between 0.9 and 1.1 throughout experiments. In the control group, the lower part of the P-vs-t curve was also linear, indicating no variation of compliance at the beginning of inspiration; values of $b_{\text {lower }}$ were also situated between 0.9 and 1.1 in this group.

The P-vs-t relation at the upper portion of the curve was linear at baseline, indicating that the compliance was constant in the upper $\mathrm{VT}$ range used before randomization (Fig. 2). In the nonprotective OLV group, the upper portion of the P-vs-t curve showed an upward concavity, indicating a progressive decrease in compliance at the higher VT range; values of $b_{\text {upper }}$ were situated between 1.43 and 2.10 after randomization. In the protective OLV group, the upper portion of the P-vs-t curve was linear, indicating a constant compliance at middle and end-inspiration; values of $b_{\text {upper }}$ were situated between 0.9 and 1.1 throughout experiments. In the control group, the lower part of the P-vs-t curve was linear, indicating a constant compliance at middle and end-inspiration; values of $b_{\text {upper }}$ were also situated between 0.9 and 1.1.

The peak inspiratory pressure (PIP) levels achieved in the nonprotective OLV group were higher than those in the protective OLV and control groups $(P<$ 0.001) (Fig. 2). In addition, the PIP level increased significantly with time in the nonprotective OLV group, as compared with Time $0(P<0.01)$. No statistically significant differences were observed between the protective OLV and control groups. Also, there was no significant increase of PIP with time in the protective OLV and control groups.

We found no statistically significant differences in mean MPAP at baseline among animals (Fig. 3). A slight but significant increase in MPAP (2 and $1 \mathrm{~mm}$ $\mathrm{Hg}$ on average, respectively) was observed in both the nonprotective and protective OLV groups when OLV was initiated, compared with baseline. In the nonprotective OLV group, but not in the protective OLV group, MPAP increased further compared with the control group, achieving levels as high as $90 \mathrm{~mm} \mathrm{Hg}$ at $90 \mathrm{~min}$. There were no statistically significant differences in MPAP between the protective OLV and control groups at the end of experiments.

There were no statistically significant differences in WG between the control and protective OLV groups (Fig. 3). In addition, WG did not increase significantly in those groups during the experiments. In the nonprotective OLV group, WG was significantly higher at 
Figure 1. Representative data record illustrating the dynamic pressure versus time (P-vs-t) behavior in the experimental groups after randomization: nonprotective one-lung ventilation (top), protective one-lung ventilation (middle), and control (two-lung ventilation) (bottom). The power equations $P_{\text {Llower }}=a_{\text {lower }} \times$ $t^{\text {blower }}+c_{\text {lower }}$ and $P_{\text {Lupper }}=a_{\text {upper }} \times$ $t^{\text {bupper }}+c_{\text {upper }}$ were applied to the lower third and upper two-thirds portions of the P-vs-t curve, respectively, which are divided by vertical dashed lines; $\mathrm{P}_{\mathrm{L}}=$ transpulmonary pressure. Coefficients $b_{\text {lower }}<1$ are associated with a downward concavity, indicating alveolar collapse; coefficients $b_{\text {upper }}>1$ are associated with an upward concavity, indicating alveolar overdistension; coefficients $b_{\text {lower }}$ and $b_{\text {upper }}=1$ are associated with a straight line and indicate the absence of alveolar collapse and overdistension, respectively.

$$
P_{L, \text { lower }}=a_{\text {lower }} \times t^{b, \text { lower }}+c_{\text {lower }} \quad P_{L, \text { upper }}=a_{\text {upper }} \times t^{b, \text { upper }}+c_{\text {upper }}
$$

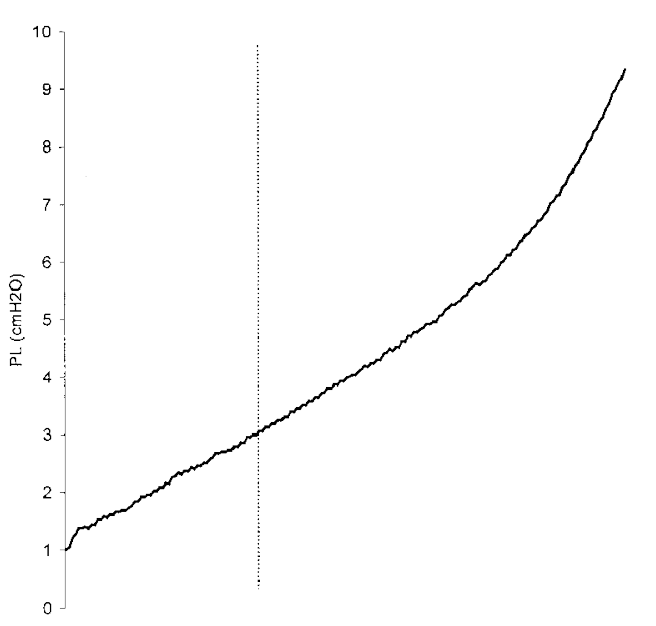

Non-protective One-Lung Ventilation

$b$, lower $=0.65$

$b$, higher $=1.71$

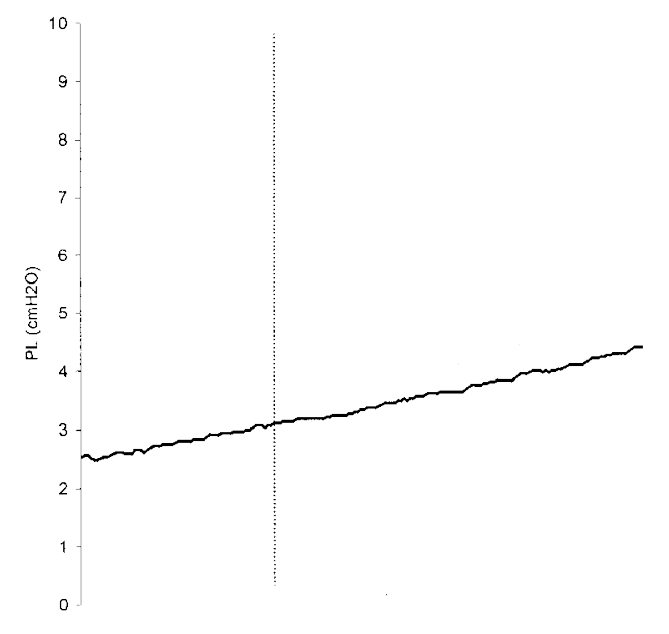

Protective One-Lung Ventilation

$b$, lower $=0.99$ $b$, higher $=1.02$

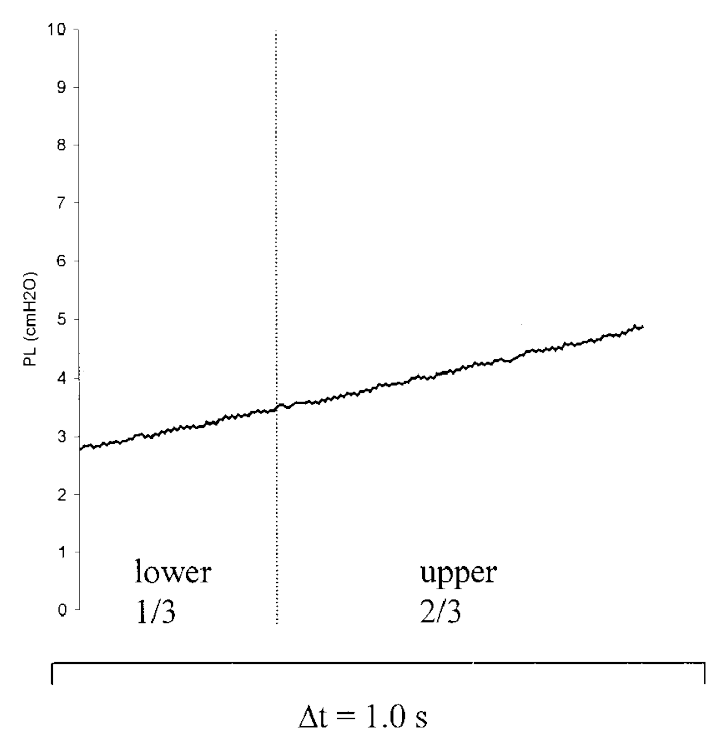

Control (Protective Two-Lungs Ventilation)

\section{$b$, lower $=0.98$ \\ $b$, higher $=1.02$}

60 and $90 \mathrm{~min}$ as compared with the protective OLV and control groups, achieving values as high as $48.8 \mathrm{~g}$.

The concentration of $\mathrm{TXB}_{2}$ in the perfusate was comparable among groups at baseline (Fig. 3). During the observation period, a significant increase of $\mathrm{TXB}_{2}$ concentration was observed in the nonprotective OLV group as compared with the protective OLV and control groups. In the protective OLV and control groups, 

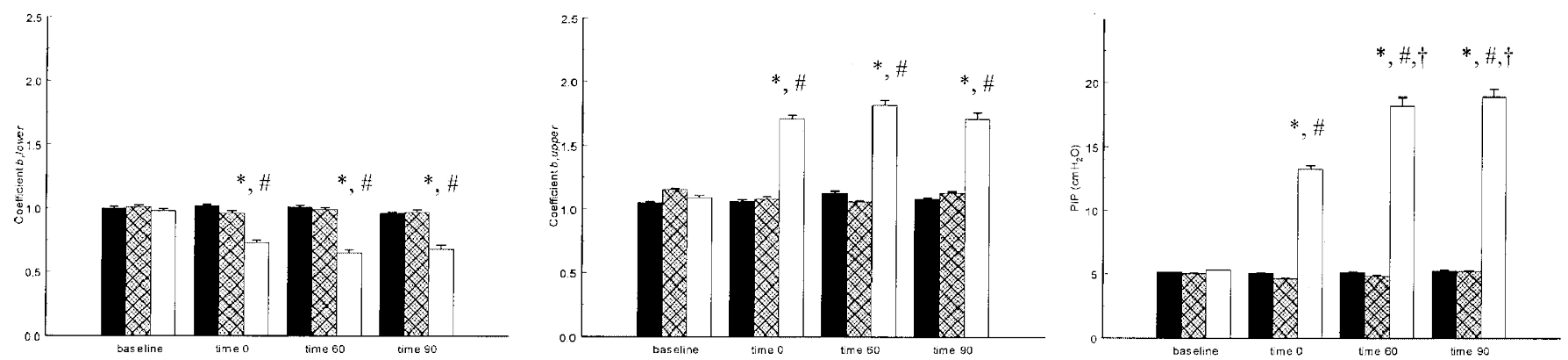

Figure 2. Values of coefficients $b_{\text {lower }}$ and $b_{\text {upper }}$ and of peak inspiratory pressure (PIP) for the different groups in the study. Black, hatched, and white bars represent the control, protective one-lung ventilation, and nonprotective one-lung ventilation groups, respectively; ${ }^{*} P<0.001$ versus control; $\# P<0.001$ versus OLV protected; $+P<0.01$ versus Time 0 .
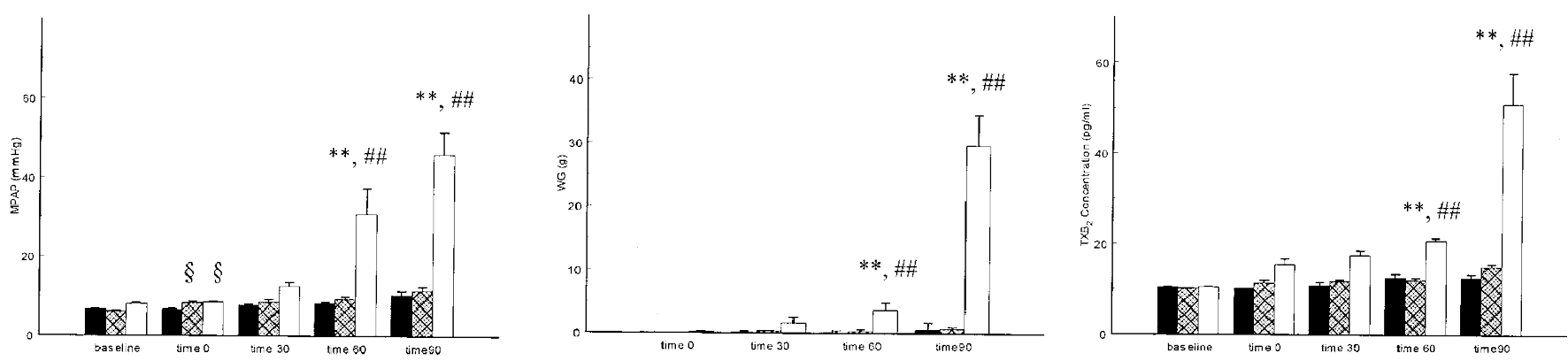

Figure 3. Values of mean pulmonary artery pressure (MPAP), lung weight gain (WG), and thromboxane $\mathrm{B}_{2}$ ( $\left(\mathrm{TXB}_{2}\right.$ ) concentration in the perfusate for the different groups in the study. Black, hatched, and white bars represent the control, protective one-lung ventilation, and nonprotective one-lung ventilation groups, respectively; ${ }^{* *} P<0.05$ versus control group; $\# \# P 0.05$ versus OLV protected; $\S P<0.01$ versus baseline.

no statistically significant increase of the concentrations of $\mathrm{TXB}_{2}$ could be observed. The $\mathrm{pH}$ of the perfusate did not differ significantly among the experimental groups during the observation period.

\section{Discussion}

One important finding of this study is that a nonprotective ventilatory strategy for OLV with VT and PEEP values similar to those used in the clinical setting has the potential to deteriorate the pulmonary mechanics, to induce pulmonary hypertension and gain of lung weight, and also to increase the release of $\mathrm{TXB}_{2}$ in the isolated, perfused rabbit lung model. Another important finding of this study is that the use of a protective strategy with VT and PEEP values set to avoid lung overdistension and collapse is able to minimize lung injury during OLV in this model.

In recent years, there has been major interest in ventilatory strategies aimed at protecting the lungs from overdistension and collapse. In patients with acute respiratory distress syndrome (ARDS), the titration of VT and PEEP according to respiratory mechanics has been suggested to be effective in improving lung function and even mortality $(11,12)$. A multicenter clinical trial on a protective ventilatory strategy for ARDS patients demonstrated that the use of reduced VT values with PEEP set according to an arterial oxygenation protocol could significantly reduce the number of ventilator-free days and hospital mortality of a group of 861 patients compared with a ventilatory strategy with high VT values (13).

Wrigge et al. (14) investigated the effect of one hour of ventilation with the protective ventilatory approach compared with a ventilatory strategy with higher VT values on the release of cytokines and pulmonary function in patients without preexisting lung injury. Those authors were not able to show a difference in the release of tumor necrosis factor (TNF)- $\alpha$, interleukin (IL)-10, IL-6, or IL-1 among groups. In addition, the pulmonary function did not deteriorate in the group with nonprotective ventilation. Those findings, however, may not be valid if ventilation is performed for longer periods of time or the distribution of ventilation is importantly altered, as may be observed during OLV.

The best way to set an optimal mechanical ventilatory strategy to minimize the risk of ventilatorassociated lung injury has been subject of intense debate (15). In this study, the mechanical ventilator settings were defined by using the dynamic characteristic of the P-vs-t curve of the respiratory system. This 
method can predict a minimally injurious ventilatory strategy in an ARDS model (10) and also in patients with ARDS (16), and it seems to be useful for the purpose of defining individual mechanical ventilatory settings. Because the P-vs-t curve had a sigmoidal shape in our study, we analyzed both the lower and the upper portions by means of the power equation: $P_{\mathrm{L}}=a \times t^{b}+c$. Therefore, we implicitly assumed that $P_{\mathrm{L}}$ should vary linearly with time (volume) during the entire inspiratory cycle if collapse and overdistension could be avoided. Using the targets recommended by Ranieri et al. (10), we defined the ventilatory settings of the protective OLV and control groups to obtain $b_{\text {lower }}$ and $b_{\text {upper }}$ coefficients approximately equal to the unit and were able to minimize VILI in those groups. Conversely, in the nonprotective OLV group, $b_{\text {lower }}$ coefficients were much less than one, whereas $b_{\text {upper }}$ coefficients were much higher than the unit. Whereas $b_{\text {lower }}$ coefficients $<0.9$ are associated with collapse, $b_{\text {upper }}$ coefficients $>1.1$ are associated with overdistension of alveoli (10).

The development of VILI in the nonprotective OLV group was mirrored by a significant increase in MPAP and WG. The increase of pulmonary vascular resistance is one of the hallmarks of lung injury of different causes (17). In the in vivo situation, the increase of MPAP usually correlates with the degree of hypoxemia and the protective hypoxic vasoconstrictory effect (18). In the isolated rabbit lung model, the partial pressure of oxygen in the perfusate is practically the same as in the atmosphere, and there is virtually no exchange in the alveoli. Thus, the MPAP increase is most probably explained by the release of inflammatory mediators. Indeed, the concentration of $\mathrm{TXB}_{2}$, the stable metabolite of $\mathrm{TXA}_{2}$, was much larger in the nonprotective OLV group. TXA 2 is an inflammatory mediator that has been implicated in the genesis of lung injury $(19,20)$. Besides the potent pulmonary vasoconstrictory property, this metabolite may enhance the extravasation of fluid into the structurally damaged lung parenchyma, with a consequent increase of lung weight and stiffness. The increase in lung stiffness is usually accompanied by the increase in airway pressures needed to achieve a given volume. This phenomenon was also present in the nonprotective OLV group, as evidenced by a progressive increase of PIP over time.

Alveoli overdistension and shear stress disrupt the pulmonary epithelium, leading to microvascular leakage and pulmonary edema (7). In addition, structural lung disruption caused by the use of high VT values is associated with the inhibition of the Na,K-ATPase function of alveolar Type II cells and impairment of the ability to clear edema (21). A link between mechanical stress and release of inflammatory mediators has been demonstrated in different studies. Investigators showed that membrane stretch may lead to disruption, activation of ion channels, and conformational changes in the structure of integrins $(22,23)$. All these mechanisms lead to an increase of gene expression, with production of cytokines and pro- and antiinflammatory molecules. Although these pathophysiological mechanisms are often associated with the presence of TNF- $\alpha$ and IL- 8 in the bronchial lavage and/or plasma or perfusate, Imanaka et al. (24) showed that TNF- $\alpha$ is not necessarily increased in the course of severe VILI, suggesting that different proinflammatory reactions may be involved. We determined the perfusate concentration of TNF- $\alpha$ and IL- 8 in 2 animals that had a dramatic development of VILI (nonprotective OLV) and another 2 that did not develop VILI (protective OLV and control groups) (data not shown). There was no increase of these cytokines in the course of VILI and also no difference among animals.

As expected, the presence of a nonventilated lung did not interfere with the protective effect of the ventilatory strategy with low VT and PEEP set to avoid lung collapse. In our study, a control group with ventilation of both lungs was included, and we did not observe any significant differences in the variables investigated, with the exception of MPAP, which increased only slightly when changing from two-lung ventilation to OLV in both the nonprotective and protective ventilation groups.

The isolated, perfused rabbit lung model permits the investigation of the effects of ventilatory strategies on the lungs without the influence of confounding factors, as, for instance, the metabolization of inflammatory mediators by other organ systems. However, the ventilation of one lung without the influence of the chest wall limits the extrapolation of our results to the clinical setting. When lung surgery is performed by means of thoracotomy, i.e., in the lateral decubitus position, the dependent lung is confined to a closed hemithorax, and the development of atelectasis is limited by the interaction between the lung and chest wall. Under such conditions, atelectasis may occur as the result of the paralysis of the diaphragm combined with increased intraabdominal pressure and the weight of the mediastinum and nondependent lung. Thus, the phenomenon of cycling collapse and reopening of lung zones probably occurs less with this approach. However, even if the expansion of the ventilated lung is limited in the closed hemithorax, the VT values used in the clinical setting are even greater than those used in our study. Consequently, the degree of lung expansion that can be achieved during thoracotomy is at least comparable to that we obtained experimentally, provided that end-expiratory lung volumes are also comparable between those situations. We did not measure end-expiratory lung volumes in our 
study, but we visually observed that lung collapse was almost complete at end-expiration in the nonprotective OLV group. The combination of overdistension and global atelectasis probably led to an exaggeration of the mechanical stress of ventilation in this study. Therefore, our results probably represent a "worst case" scenario of OLV during thoracotomy.

Another important approach used for lung surgery is the median sternotomy, which may be superior to the thoracotomy for certain procedures $(25,26)$. This approach is frequently seen during lung volume reduction surgery $(27,28)$ and may be advantageous for resection of lung metastases $(29,30)$. Usually, both hemithoraxes are open to the atmosphere during lung surgery with the median sternotomy approach, and the ventilated lung may be able to expand and collapse with minimal interaction with the chest wall. Because the lung mechanics during such an approach are similar to those observed in our model, our results suggest that the conventional, nonprotective ventilatory strategy for OLV should be avoided during median sternotomy.

The reexpansion of the lungs after longer periods of collapse is associated with increased concentrations of inflammatory mediators in plasma (31), which may contribute to the development of postoperative lung injury and even infection. We were concerned about those issues when we performed our study. However, the nonprotective ventilatory strategy led to dramatic degrees of lung injury, which would have not allowed the investigation of the effects of reexpansion of the nonventilated lungs. Thus, interpretation of our findings must take into account that only the OLV phase was addressed. No controlled trials have been performed to investigate the possible harmful effects of the nonprotective ventilatory strategy with high VT values and zero PEEP during OLV under different surgical conditions. Thus, clinical studies are necessary to confirm our findings.

We are indebted to Prof. P. Dieter, MD, PhD, and M. Kolada (Institute of Biochemistry, University Clinic Carl Gustav Carus, Dresden, Germany) for the measurement of $\mathrm{TXB}_{2}$. We also wish to thank E. Kuhlisch (Institute of Biometry, University Clinic Carl Gustav Carus, Dresden, Germany) for assistance in statistics.

\section{References}

1. Ribas J, Jimenez MJ, Barbera JA, et al. Gas exchange and pulmonary hemodynamics during lung resection in patients at increased risk: relationship with preoperative exercise testing. Chest 2001;120:852-9.

2. Brodsky JB, Fitzmaurice B. Modern anesthetic techniques for thoracic operations. World J Surg 2001;25:162-6.

3. Inomata S, Nishikawa T, Saito S, et al. "Best" PEEP during one-lung ventilation Br J Anaesth 1997;78:754-6.
4. Slinger PD, Kruger M, McRae K, et al. Relation of the static compliance curve and positive end-expiratory pressure to oxygenation during one-lung ventilation. Anesthesiology 2001;95: 1096-102.

5. Dreyfuss D, Soler P, Saumon G. Mechanical ventilation-induced pulmonary edema: interaction with previous lung alterations. Am J Respir Crit Care Med 1995;151:1568-75.

6. Broccard AF, Hotchkiss JR, Suzuki S, et al. Effects of mean airway pressure and tidal excursion on lung injury induced by mechanical ventilation in an isolated perfused rabbit lung model. Crit Care Med 1999;27:1533-41.

7. Dreyfuss D, Saumon G. Ventilator-induced lung injury: lessons from experimental studies. Am J Respir Crit Care Med 1998;157: 294-323.

8. Koch T, Duncker HP, Rosenkranz S, et al. Alterations of filtration coefficients in pulmonary edema of different pathogenesis. J Appl Physiol 1992;73:2396-402.

9. Bates JHT, Rossi A, Milic-Emili J. Analysis of the behavior of the respiratory system with constant respiratory flow. J Appl Physiol 1985;58:1840-8.

10. Ranieri VM, Zhang H, Mascia L, et al. Pressure-time curve predicts minimally injurious ventilatory strategy in an isolated rat lung model. Anesthesiology 2000;93:1320-8.

11. Amato MBP, Barbas CSV, Medeiros DM, et al. Beneficial effects of the "open lung approach" with low distending pressures in acute respiratory distress syndrome: a prospective randomized study on mechanical ventilation. Am J Respir Crit Care Med 1995;152:1835-46.

12. Amato MBP, Barbas CSV, Medeiros DM, et al. Effect of a protective-ventilation strategy on mortality in the acute respiratory distress syndrome. N Engl J Med 1998;338:347-54.

13. The Acute Respiratory Distress Syndrome Network. Ventilation with lower tidal volumes as compared with traditional tidal volumes for acute lung injury and the acute respiratory distress syndrome. N Engl J Med 2000;342:1301-8.

14. Wrigge H, Zinserling J, Stuber F, et al. Effects of mechanical ventilation on release of cytokines into systemic circulation in patients with normal pulmonary function. Anesthesiology 2000; 93:1413-7.

15. International consensus conferences in intensive care medicine: ventilator-associated lung injury in ARDS. Intensive Care Med $1999 ; 25: 1444-52$.

16. Ranieri VM, Giuliani R, Fiore T, et al. Volume-pressure curve of the respiratory system predicts effects of PEEP in ARDS: "occlusion" versus "constant flow" technique. Am J Respir Crit Care Med 1994;149:19-27.

17. Gattinoni L, Pelosi P, Suter PM, et al. Acute respiratory distress syndrome caused by pulmonary and extrapulmonary disease: different syndromes? Am J Respir Crit Care Med 1998;158:3-11.

18. Hales CA, Westphal DM. Pulmonary hypoxic vasoconstriction: not affected by chemical sympathectomy. J Appl Physiol 1979; 46:529-33.

19. Gust R, Kozlowski JK, Stephenson AH, et al. Role of cyclooxygenase-2 in oleic acid-induced acute lung injury. Am J Respir Crit Care Med 1999;160:1165-70.

20. Schmeck J, Janzen R, Munter K, et al. Endothelin and thromboxane $\mathrm{A}_{2}$ increase pulmonary vascular resistance in granulocyte-mediated lung injury. Crit Care Med 1998;26: $1868-74$.

21. Lecuona E, Saldías F, Comellas A, et al. Ventilator-associated lung injury decreases lung ability to clear edema in rats. Am J Respir Crit Care Med 1999;159:603-9.

22. Tremblay L, Valenza F, Ribeiro SP, et al. Injurious ventilatory strategies increase cytokines and c-fos m-RNA expression in an isolated rat lung model. J Clin Invest 1997;99:944-52.

23. Gembrowicz KP, Sprague D, McNeil PL. Temporary disruption of the plasma membrane is required for c-fos expression in response to mechanical stress. Mol Biol Cell 1999;10:1247-57.

24. Imanaka H, Shimaoka M, Matsuura N, et al. Ventilator-induced lung injury is associated with neutrophil infiltration, macrophage activation, and TGF- $\beta 1$ mRNA upregulation in rat lungs. Anesth Analg 2001;92:428-36. 
25. Asaph JW, Handy JR Jr, Grunkemeier GL, et al. Median sternotomy versus thoracotomy to resect primary lung cancer: analysis of 815 cases. Ann Thorac Surg 2000;70:373-9.

26. Urschel HC Jr, Razzuk MA. Median sternotomy as a standard approach for pulmonary resection. Ann Thorac Surg 1986;41: $130-4$.

27. Eugene J, Dajee A, Kayaleh R, et al. Reduction pneumonoplasty for patients with a forced expiratory volume in 1 second of 500 milliliters or less. Ann Thorac Surg 1997;63:186-90.

28. Miller JI Jr, Lee RB, Mansour KA. Lung volume reduction surgery: lessons learned. Ann Thorac Surg 1996;61:1464-8.
29. Rusch VW. Pulmonary metastasectomy: current indications. Chest 1995;107:322S-31.

30. van der Veen AH, van Geel AN, Hop WC, et al. Median sternotomy: the preferred incision for resection of lung metastases. Eur J Surg 1998;164:507-12.

31. Yamada T, Hisanaga M, Nakajima Y, et al. Serum interleukin-6, interleukin-8, hepatocyte growth factor, and nitric oxide changes during thoracic surgery. World J Surg 1998;22:783-90. 